\title{
Inverted Pendulum-type Personal Mobility Considering Human Vibration Sensitivity
}

\author{
Misaki Masuda \\ School of Science for Open and Environmental Systems \\ Keio University \\ Yokohama, Japan
}

Kazuto Yokoyama

School of Science for Open and Environmental Systems

Keio University

Yokohama, Japan

\author{
Takuma Suzuki \\ Graduate School of Science and Technology \\ Keio University \\ Yokohama, Japan \\ Masaki Takahashi \\ Department of System Design Engineering \\ Keio University \\ Yokohama, Japan
}

\begin{abstract}
An inverted pendulum-type PM (personal mobility) has been attracting attention as a low-carbon vehicle. For many people who like to use the PM, ride comfort is important. However, ride comfort of PM has not been focused on in previous studies. The vibration is one of causes that make riders feel uncomfortable. The PM is unstable system and horizontal vibration may be caused by a stabilizing control. Additionally, vertical vibration may also be caused by road disturbances. This study analyzes the vibration of the rider's head in these two directions when the PM runs on a road with disturbances in numerical simulations, and evaluates ride comfort with the frequency characteristics of the vibration. To consider human vibration sensitivity, the frequency weighting proposed in ISO 2631-1 is used as an evaluation standard. The improvement methods are proposed from both software and hardware, and it is confirmed that the proposed method can improve ride comfort.
\end{abstract}

Keywords-Inverted Pendulum-Type Personal Mobility; Ride Comfort; Human Vibration; Frequency Analysis; Vibration Control; Frequency Shaped LQG

\section{INTRODUCTION}

Recently in Japan, the number of single-person households has increased through declining birthrate and a rapidly growing proportion of elderly people, and the population decreases [1]. Based on this current situation, the PM has been attracting attention as a next-generation mobility vehicle. The PM is a vehicle for personal short-distance trips, and it is an electric vehicle. The single-person household using a PM instead of a gasoline vehicle is expected to contribute to the low-carbon society. Studies of dynamics and stability of the PM have been conducted [2]. Studies of model and control characteristics of human on the PM have been recently conducted [3][4]. Many stabilization control methods for the PM have been proposed [5]-[8]. It is important to improve ride comfort so that many people use the PM. However, the ride comfort problem has not been focused on in previous studies.
This paper aims at improving ride comfort of the PM. The model that was studied was an inverted pendulum PM that has many advantages and is widely used. The system of the inverted pendulum PM is unstable. Therefore, horizontal vibration occurs by a stabilizing control. Additionally, vertical vibration occurs when traveling due to road disturbances. This study analyzes the vibration in these two directions and evaluates ride comfort of the occupant considering the human vibration sensitivity. This study proposes an inverted pendulum PM with greater ride comfort using both hardware and software approaches based on the analysis results. To verify its effectiveness, numerical simulations were carried out.

\section{MODELING}

\section{A. Model and Parameter}

Figs. 1 (A), (B) and (C) show a coordinate system, a model for numerical simulation, and a model for design of control system respectively. In our study, the models of an inverted pendulum-type PM are limited to two-dimension, horizontal and vertical.

The following variables have been chosen to describe the vehicle (see also Fig. 1):

- $\theta$ : Pendulum angle with respect to $\mathrm{z}$ axis.

- $\varphi$ : Tire angle with respect to pendulum angle.

- $u$ : Control input torque.

- $d$ : Displacement of road disturbance.

Parameter definitions and values are collected in TABLE I. Parameters of the vehicle are referring to Segway PT i2 [9]. The occupant was assumed that the height is $1.70 \mathrm{~m}$, and weight is $65.0 \mathrm{~kg}$. This refers to average height and weight of Japanese adult male [10]. Tire stiffness and damping coefficient are referring to the study of Sharp et al [11]. 


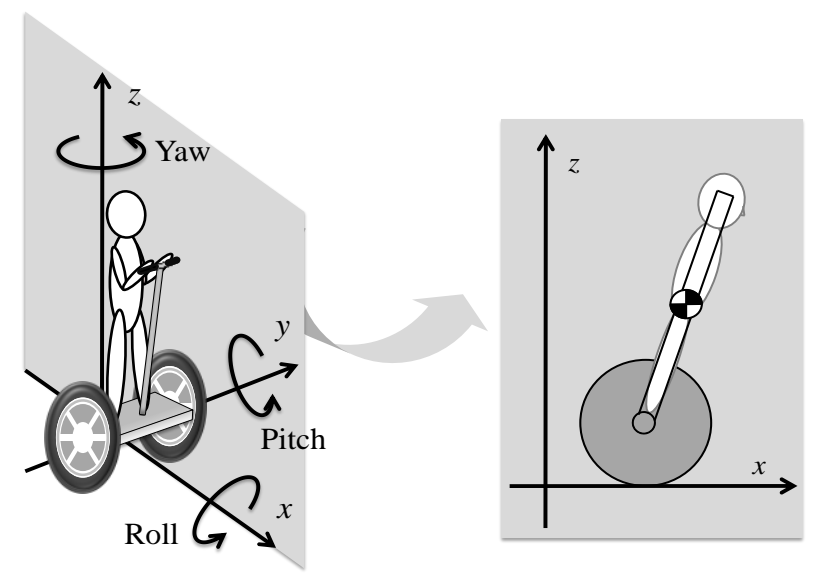

(A) Coordinate system

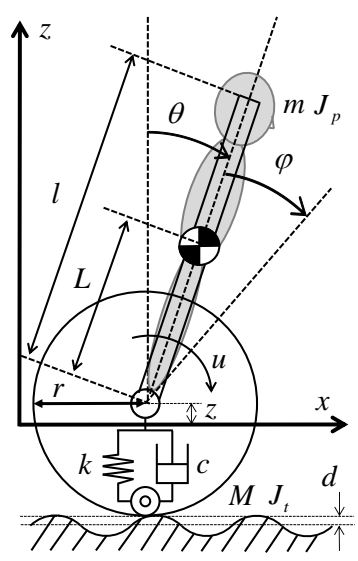

(B) A model for simulation

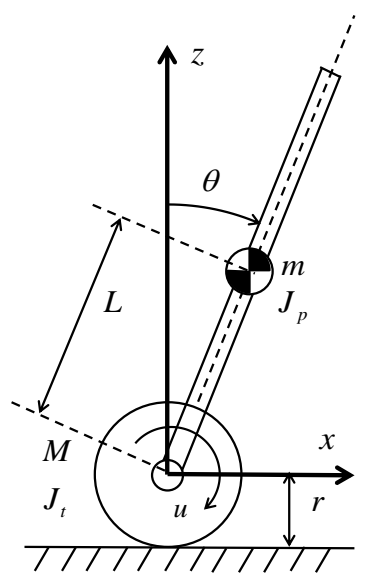

(C) A model for control system design
Fig. 1. Diagrams of an inverted pendulum-type personal mobility

\section{B. Motion Equations of Model for Numerical Simulation}

Coordinates of the center of the tire $\left(x_{t}, z_{t}\right)$, and of the center of gravity of the pendulum $\left(x_{p}, z_{p}\right)$ are

$$
\begin{gathered}
\left(x_{t}, z_{t}\right)=(r(\theta+\varphi), z) \\
\left(x_{p}, z_{p}\right)=\left(x_{t}+L \sin \theta, z_{t}+L \cos \theta\right)
\end{gathered}
$$

In Fig. 1(B), kinetic energy $T$, potential energy $V$, and dissipation energy $D$ of the numerical simulation model are

$$
\left\{\begin{array}{l}
T=\frac{1}{2} M\left(\dot{x}_{t}^{2}+\dot{z}_{t}^{2}\right)+\frac{1}{2} m\left(\dot{x}_{p}^{2}+\dot{z}_{p}^{2}\right)+\frac{1}{2} J_{p} \dot{\theta}^{2}+\frac{1}{2} J_{t}(\dot{\theta}+\dot{\varphi})^{2} \\
V=m g L \cos \theta+\frac{1}{2} \cdot 2 k(z-d)^{2}=m g L \cos \theta+k(z-d)^{2} \\
D=\frac{1}{2} \cdot 2 c(\dot{z}-\dot{d})^{2}=c(\dot{z}-\dot{d})^{2}
\end{array}\right.
$$

TABLE I. MODEL PARAMETERS

\begin{tabular}{|l|c|c|c|}
\hline \multicolumn{1}{|c|}{ Parameter } & Symbol & Unit & Value \\
\hline Mass of the pendulum & $m$ & $\mathrm{~kg}$ & 103.0 \\
\hline Mass of the tires & $M$ & $\mathrm{~kg}$ & 10.0 \\
\hline Moment of inertia of the pendulum & $J_{p}$ & $\mathrm{kgm}^{2}$ & 21.7 \\
\hline Moment of inertia of the tire & $J_{t}$ & $\mathrm{kgm}^{2}$ & 0.29 \\
\hline Height of the occupant & $l$ & $\mathrm{~m}$ & 1.70 \\
\hline $\begin{array}{l}\text { Distance between the tire center and } \\
\text { center of gravity of the pendulum }\end{array}$ & $L$ & $\mathrm{~m}$ & 0.70 \\
\hline Radius of the tire & $r$ & $\mathrm{~m}$ & 0.24 \\
\hline Tire stiffness & $k$ & $\mathrm{~N} / \mathrm{m}$ & $1.0 \times 10^{5}$ \\
\hline Tire damping coefficient & $c$ & $\mathrm{~kg} / \mathrm{s}$ & $1.0 \times 10^{2}$ \\
\hline
\end{tabular}

By using the Lagrangian, the following motion equations are obtained:

$$
\begin{gathered}
\left\{(M+m) r^{2}+2 m r L \cos \theta+m L^{2}+J_{p}+J_{t}\right\} \ddot{\theta} \\
+\left\{(M+m) r^{2}+m r L \cos \theta+J_{t}\right\} \ddot{\varphi}-m L \sin \theta \ddot{z} \\
-m r L \dot{\theta}^{2} \sin \theta-m g L \sin \theta=0 \\
\left\{(M+m) r^{2}+m r L \cos \theta+J_{t}\right\} \ddot{\theta}+\left\{(M+m) r^{2}+J_{t}\right\} \ddot{\varphi} \\
-m r L \dot{\theta}^{2} \sin \theta=u
\end{gathered}
$$

$$
\begin{aligned}
-m L \ddot{\theta} \sin \theta & +(M+m) \ddot{z}-m L \dot{\theta}^{2} \cos \theta \\
& +2 k(z-d)+2 c(\dot{z}-\dot{d})=0
\end{aligned}
$$

\section{DESIGN OF CONTROL SYSTEM}

\section{A. Motion Equations of the Model for Control System}

In Fig. 1(C), the following motion equations are obtained as

$$
\begin{aligned}
& \left\{(M+m) r^{2}+2 m r L \cos \theta+m L^{2}+J_{p}+J_{t}\right\} \ddot{\theta} \\
& +\left\{(M+m) r^{2}+m r L \cos \theta+J_{t}\right\} \ddot{\varphi}-m r L \dot{\theta}^{2} \sin \theta \\
& -m g L \sin \theta=0
\end{aligned}
$$

$$
\begin{array}{r}
\left\{(M+m) r^{2}+m r L \cos \theta+J_{t}\right\} \ddot{\theta}+\left\{(M+m) r^{2}+J_{t}\right\} \ddot{\varphi} \\
-m r L \dot{\theta}^{2} \sin \theta=u
\end{array}
$$

\section{B. State-Space Representation}

$\theta$ is assumed to be near 0 , therefore $\sin \theta \approx \theta, \cos \theta \approx 1$, $\dot{\theta}^{2} \approx 0$ are assumed. On this assumption, the equations are linearly approximated, and represented as the following statespace. 


$$
\dot{\mathbf{x}}_{c}=\mathbf{A} \mathbf{x}_{c}+\mathbf{B} u
$$

The control target vector is $\mathbf{x}_{c}=\left[\begin{array}{ll}\theta & \dot{\theta}\end{array}\right]^{T}$.

\section{Design of Optimal Regulator}

The design of the optimal regulator has done using three gains. We call them Controller 1, Controller 2, and Controller 3 respectively. TABLE II shows the feedback gains, $\mathbf{K}=\left[\begin{array}{ll}K_{\theta} & K_{\dot{\theta}}\end{array}\right]$, and the settling time of $\theta$ of each controller. Control input torque is $u=-\mathbf{K} \mathbf{x}_{c}$. The settling time of $\theta$ is the time that $\theta$ has been less than $1.0 \times 10^{-4} \mathrm{rad}$.

As shown in TABLE II, the controllers have different settling times. Settling time of each controller is relatively slow, medium, and fast.

\section{CONDITIONS OF SIMULATION AND ANALYSIS}

\section{A. Initial State}

State variable vector for numerical simulation model is defined as

$$
\mathbf{x}=\left[\begin{array}{llllll}
\theta & \varphi & z & \dot{\theta} & \dot{\varphi} & \dot{z}
\end{array}\right]^{T}
$$

The initial state vector is

$$
\mathbf{x}_{\mathbf{0}}=\left[\begin{array}{llllll}
0.017 & 0 & 0 & 0 & 23.1 & 0
\end{array}\right]^{T}
$$

\section{B. Road Disturbance}

There are two types of road disturbance. One is sinusoidal, and the other one is random.

\section{1) Sinusoidal road: The sinusoidal road is defined as}

$$
d=a \sin \omega b x
$$

$a$ is amplitude of the disturbance, and $a=0.005 \mathrm{~m} . b$ is a conversion factor. When the PM is traveling at desired speed (in this paper, $5.6 \mathrm{~m} / \mathrm{s}$ ), $b=0.180$ so as to satisfy $\omega b x=\omega t$. When $\omega=2 \pi f$ is substituted to Eq. (12), Eq. (12) is assumed to be equivalent to the sinusoidal road displacement whose frequency is $f \mathrm{~Hz}$. In this paper, the frequency is 7 pattern, 0.1 , $0.5,1.0,5.0,10.0,16.0$, and $20.0 \mathrm{~Hz}$.

2) Random road: The design of random road is refer to ISO 8608 [12]. In this paper, classification of road is Level A, $B, C, D$, and E. Each level means that the road is very good, good, normal, poor, and very poor.

\section{Target of Analysis}

Coordinates of the head of the occupant $\left(x_{h}, z_{h}\right)$ is

$$
\left(x_{h}, z_{h}\right)=\left(x_{t}+l \sin \theta, z_{t}+l \cos \theta\right)
$$

TABLE II. PROPERTIES OF CONTROLLERS

\begin{tabular}{|l|c|c|c|c|c|}
\hline & \multirow{2}{*}{ Symbol } & \multirow{2}{*}{ Unit } & \multicolumn{3}{|c|}{ Controller } \\
\cline { 4 - 6 } & & & $\mathbf{1}$ & $\mathbf{2}$ & $\mathbf{3}$ \\
\hline $\begin{array}{l}\text { Feedback } \\
\text { gains }\end{array}$ & $K_{\theta}$ & $\mathrm{Nm} / \mathrm{rad}$ & -398.5 & -404.7 & -573.0 \\
\cline { 2 - 6 } & $K_{\dot{\theta}}$ & $\mathrm{Nm} / \mathrm{rad}$ & -94.0 & -80.1 & -95.3 \\
\hline Settling time & $T$ & $\mathrm{~s}$ & 2.00 & 1.40 & 0.73 \\
\hline
\end{tabular}

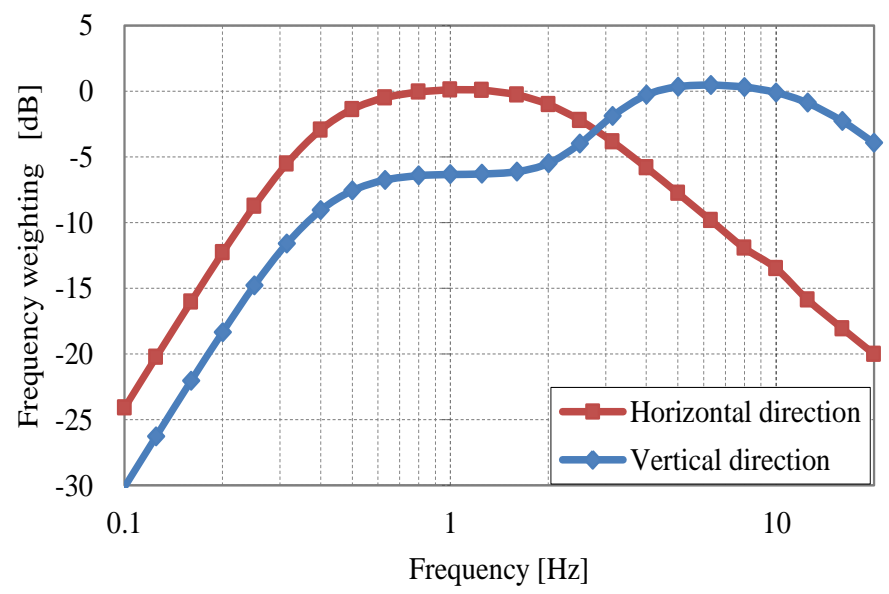

Fig. 2. Frequency weighting curves shown in ISO 2631-1

Acceleration of the head of the occupant is

1)

$$
\left\{\begin{array}{l}
\ddot{x}_{h}=\ddot{x}_{t}+l \ddot{\theta} \cos \theta-l \dot{\theta}^{2} \sin \theta \\
\ddot{z}_{h}=\ddot{z}_{t}-l \ddot{\theta} \sin \theta-l \dot{\theta}^{2} \cos \theta
\end{array}\right.
$$

\section{Evaluation Method}

In this paper, Power Spectrum Density (PSD) and Root Mean Square (RMS) are used for evaluation.

1) PSD: PSD is evaluated by using the frequency weighting curves that are proposed in ISO 2631-1 [13], as

2) shown in Fig. 2. Fig. 2 shows that the unpleasant frequency range is about $0.8-1.6 \mathrm{~Hz}$ in the horizontal direction and $4.0-8.0 \mathrm{~Hz}$ in the vertical direction.

3) RMS: RMS is defined [13]:

$$
a_{w}=\left[\frac{1}{T} \int_{0}^{T} a_{w}^{2}(t) d t\right]^{\frac{1}{2}}
$$

$T$ is simulation time, in this paper, $T=30 \mathrm{~s}$. The acceleration of the head of occupant is substituted to $a_{w}(t)$. The RMS calculated by Eq. (15) does not reflect human vibration sensitivity. Therefore, the acceleration data is filtered to consider human vibration sensitivity. The transfer function representation of the frequency weighting proposed in ISO2631-1 is used as a filter [14]. The transfer functions are 


$$
\left\{\begin{array}{l}
W_{d}(s)=\frac{12.66 s^{3}+163.7 s^{2}+60.64 s+12.79}{s^{4}+23.77 s^{3}+236.1 s^{2}+692.8 s+983.4} \\
W_{k}(s)=\frac{80.03 s^{2}+989.0 s+0.02108}{s^{3}+78.92 s^{2}+2412 s+5614}
\end{array}\right.
$$

$W_{d}$ is the transfer function in the horizontal direction, and $W_{k}$ is that in the vertical direction.

\section{EVALUATION OF RIDE COMFORT}

Fig. 3 shows a rider's head acceleration when the road disturbance is sinusoidal $(f=1.0 \mathrm{~Hz})$. Figs. 4 and 5 show the PSD and RMS of the head acceleration, respectively. In the horizontal direction, the result is different due to changing the controller, as shown in Fig, 3(A). Figs. 4(A) and 5(A) show that the faster the settling time of the controller is, the better ride comfort become. However when Controller 3, whose settling time is the fastest of the three controllers, is used, the ride comfort gets worse. It is confirmed that if the settling time is less than $1.2 \mathrm{~s}$, the ride comfort gets worse. Therefore, controller whose settling time is too fast, for example, Controller 3, is undesirable as a control system considering the ride comfort. In the vertical direction, the results are almost the same even if the controllers are changed, as shown in Fig. 3(B). The same trends can be seen in Figs. 4(B) and 5(B). This suggests that improving on the control system design is difficult. In addition, in Fig. 4(B), there are two peaks of PSD. One is the frequency of the vibration that is included in the road disturbance. The other is $6.7 \mathrm{~Hz}$ on any roads. The frequency, that is the natural frequency of this model, is in the frequency range that human feels uncomfortable in the vertical direction $(4.0-8.0 \mathrm{~Hz})$. Therefore, in the vertical direction, it is required to change the natural frequency using a hardware approach.

\section{IMPROVEMENT OF RIDE COMFORT}

\section{A. Proposal for Improving Ride Comfort}

. Improvement Method in the Horizontal Direction: When the optimal regulator controllers are used, the faster the settling time of the controller is, the better ride comfort become. However, if the settling time is too fast, ride comfort gets worse. Therefore we apply a frequency shaping LinearQuadratic-Gaussian (LQG) controller [15] to control PM as an approach from the control system. Using this method, it isintended to reduce the vibration in the frequency range $(0.8$ $1.6 \mathrm{~Hz}$ ), while keeping the settling time of $\theta$ about $1.40 \mathrm{~s}$ which is the settling time of controller 2. Weighting on the state variable, $\mathbf{Q}(j \omega)$, and weighting on the control input, $\mathbf{R}(j \omega)$, are designed for the frequency shaping LQG control. $\mathbf{Q}(j \omega)$ is

$$
\mathbf{Q}(j \omega)=\left[\begin{array}{cc}
\mathbf{Q}_{\theta}(j \omega) & 0 \\
0 & 10^{-2}
\end{array}\right]
$$

Fig. 6 shows $\mathbf{Q}_{\theta}(j \omega)$ and $\mathbf{R}(j \omega)$. $\mathbf{Q}_{\theta}(j \omega)$ is designed to take large value in a low-frequency band up to $1.0 \mathrm{~Hz}$, and $\mathbf{R}(j \omega)$ is designed to take large value in a frequency band greater than $1.6 \mathrm{~Hz}$. These weightings represented in the transfer functions are as follows.

$$
\left\{\begin{array}{l}
\mathbf{Q}_{f \theta}(s)=\frac{118.4}{s^{2}+22.6 s+39.5} \\
\mathbf{R}_{f}(s)=\frac{63.2 s^{2}+2222.5 s+9974.7}{157.9 s^{2}+11112.6 s+99746.9}
\end{array}\right.
$$

The settling time of $\theta$ is $1.37 \mathrm{~s}$. This time is equivalent to that of the controller 2. We call this LQG controller Controller 4.

1) Improvement Method in the Vertical Direction: In the vertical direction, it is confirmed that improving on the control system design is difficult. We propose a change of the tires as a hardware approach.

The natural frequency of the PM's tire is

$$
f_{n}=\frac{1}{2 \pi} \sqrt{\frac{2 k}{M+m}}
$$

Fig. 7 shows the relation between the stiffness of the tire and the natural frequency, when the occupant weight is changed. The occupant weight is $45-118 \mathrm{~kg}$, which refers to a manual of Segway PT i2. The current tire stiffness is $1.0 \times 10^{5}$ $\mathrm{N} / \mathrm{m}$. The natural frequency is always contained in the frequency range $(4.0-8.0 \mathrm{~Hz})$ in which human feels uncomfortable. In order that the natural frequency is not included in the range, the tire stiffness needs to be more than $2.0 \times 10^{5} \mathrm{~N} / \mathrm{m}$ or less than $2.5 \times 10^{4} \mathrm{~N} / \mathrm{m}$.

It is known that the tire stiffness is dependent on the air pressure. The air pressure is dependent on the tire oblateness [16]. The tire oblateness is calculated from the tire width and height. The tire oblateness of the Segway PT i2 is 0.7. To know the tire characteristic of Segway PT i2, we refer to the tire characteristics of automobiles and motorcycles [16][17]. Automobile and motorcycle tires whose oblateness is near 0.7 are selected.

With respect to those tires, the tire stiffness and width are shown in Fig. 8. In Fig. 8, it is confirmed that the stiffness has nearly linear relationship with the tire width. Consequently, using linear interpolation of data of motorcycle tires, we obtain $k=2050 W+87000$.

The tire width can be determined from the desired tire stiffness. If the tire stiffness is more than $2.0 \times 10^{5} \mathrm{~N} / \mathrm{m}$, the tire width needs to be more than $140 \mathrm{~mm}$. In the same way, if the tire stiffness is less than $2.5 \times 10^{4} \mathrm{~N} / \mathrm{m}$, the tire width needs to be less than $55 \mathrm{~mm}$. When tire stiffness is $2.5 \times 10^{4} \mathrm{~N} / \mathrm{m}$, the RMS value is smaller than that when $200000 \mathrm{~N} / \mathrm{m}$. Therefore, we propose a tire whose width is $55 \mathrm{~mm}$. When the tire is used, the natural frequency becomes $3.4 \mathrm{~Hz}$.

\section{B. Simulation Results and Discussion}

We proposed improvement methods for each direction: horizontal and vertical. In our proposed method, the two methods are used simultaneously. 


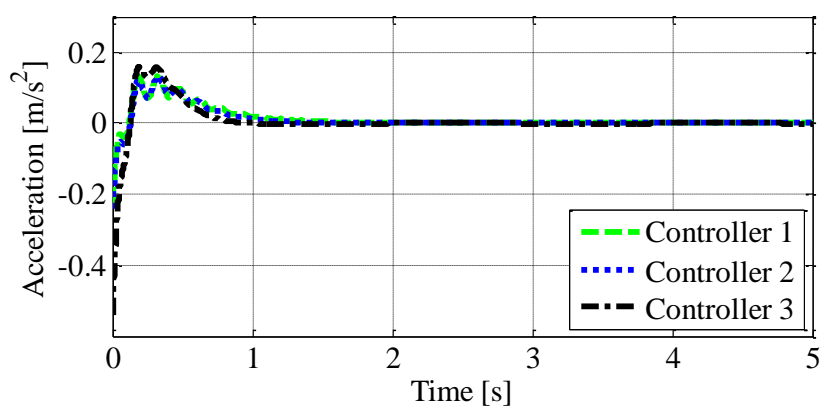

(A) Horizontal direction

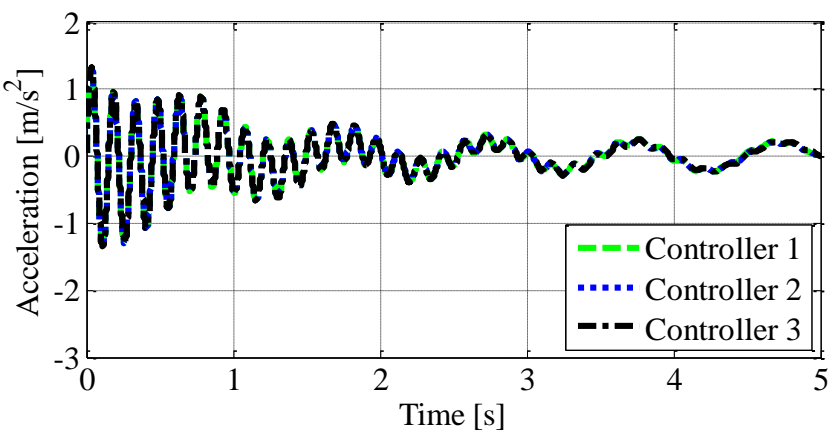

(B) Vertical direction

Fig. 3. A rider's head acceleration (Sinusoidal road, $f=1.0 \mathrm{~Hz}$ )

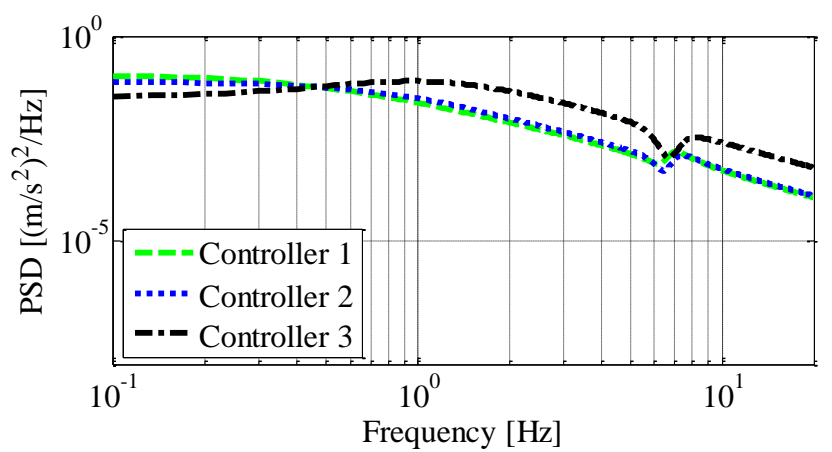

(A) Horizontal direction

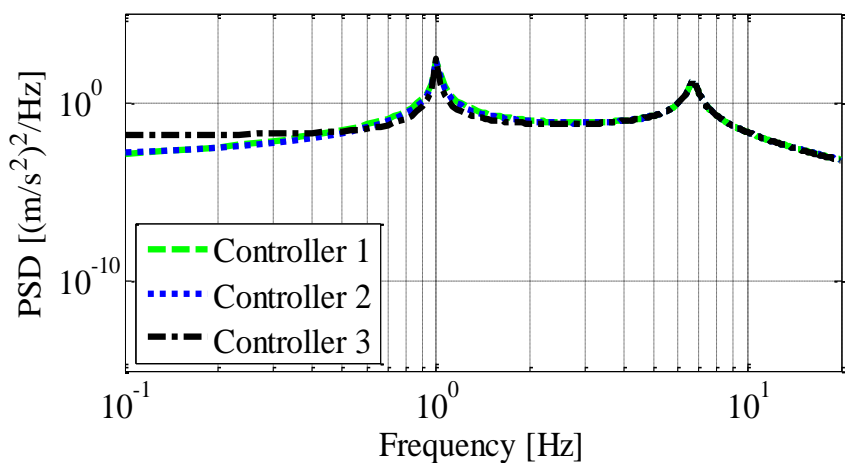

(B) Vertical direction

Fig. 4. PSD of a rider's head acceleration (Sinusoidal road, $f=1.0 \mathrm{~Hz}$ )

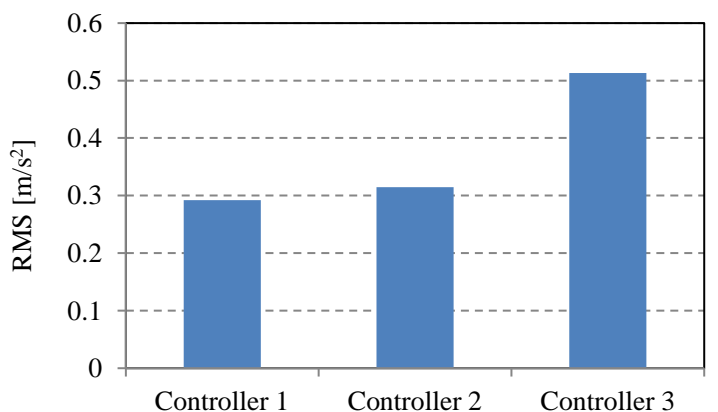

(A) Horizontal direction

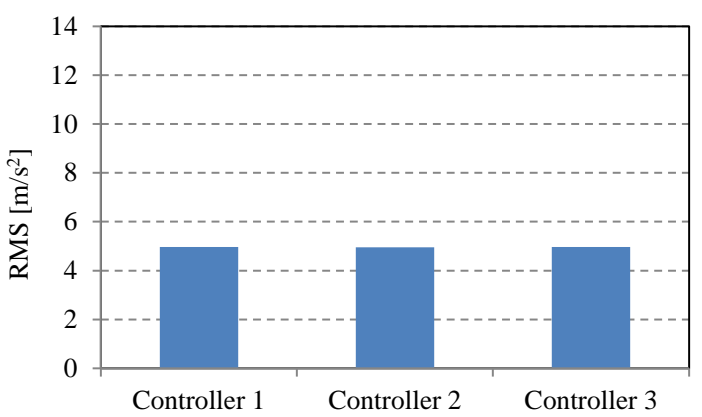

(B) Vertical direction

Fig. 5. RMS value of a rider's head acceleration (Sinusoidal road, $f$ $=1.0 \mathrm{~Hz}$ )

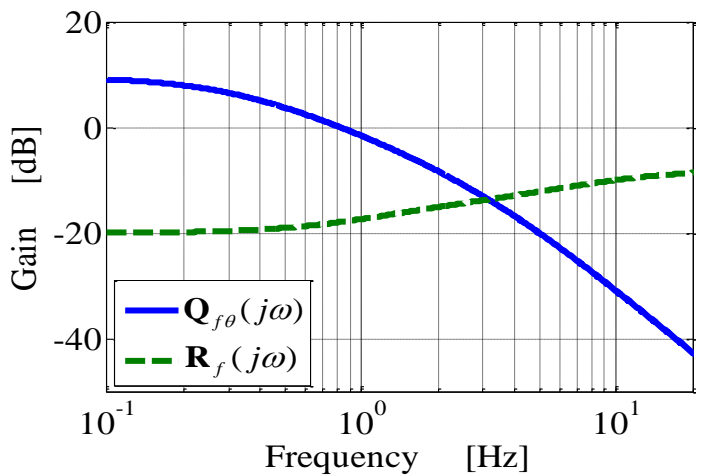

Fig. 6. Weighting functions for frequency shaped LQG

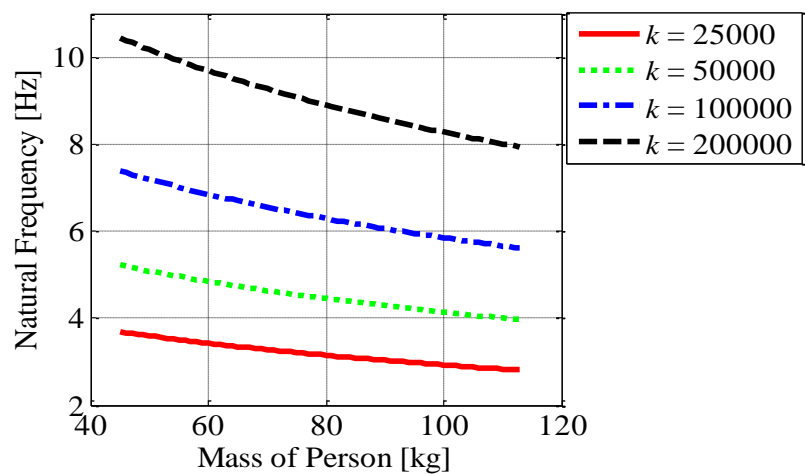

Fig. 7. Natural Frequency of the personal mobility 


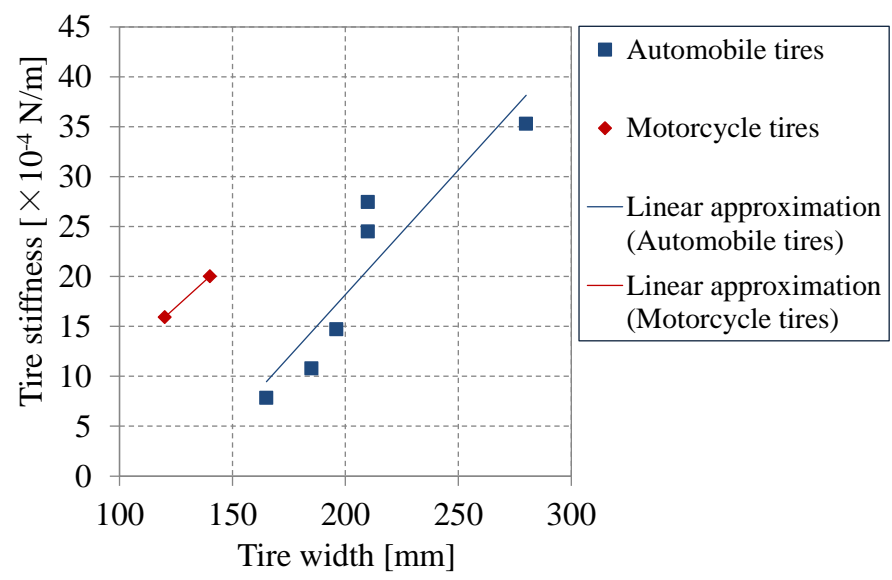

Fig. 8. Relations between the tire width and the stiffness

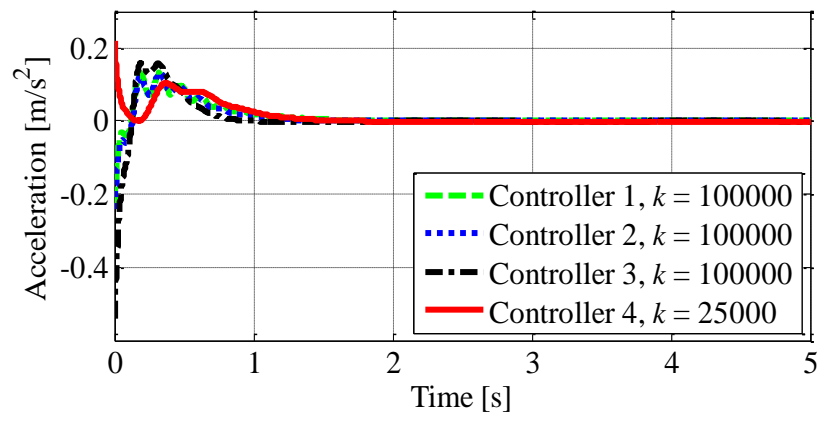

(A) Horizontal direction

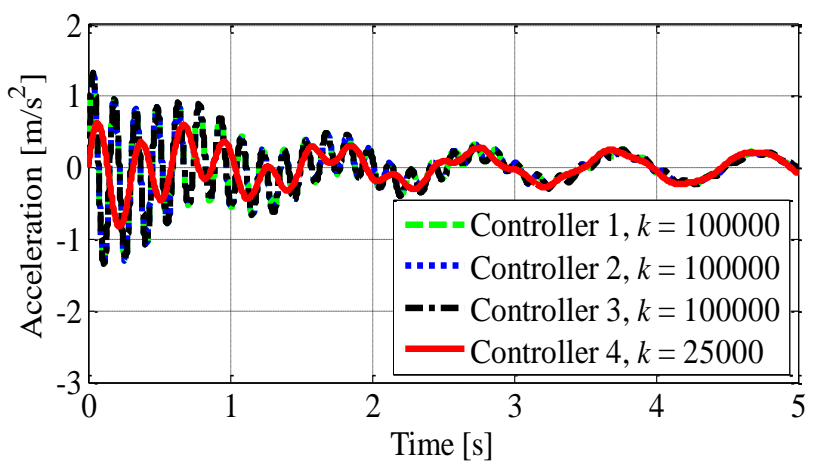

(B) Vertical direction

Fig. 9. A rider's head acceleration (Sinusoidal road, $f=1.0 \mathrm{~Hz}$ )

Fig. 9 compares rider's head acceleration of Controller 1, Controller 2, and the proposed method. The road disturbance is sinusoidal $(f=1.0 \mathrm{~Hz})$. Fig. 10 and 11 show the results when the road disturbance is random (Level C) which simulate the actual road. Fig. 10 shows PSD of the head acceleration and Fig. 11 shows RMS of it. Based on the results in Chapter V, only Controller 1 and 2 are used in simulation for verification.

In Fig. 9(A), the proposed method does not modify the maximum value of the horizontal head acceleration. In Fig. 10(A), where the road disturbance is random, the vibration in the uncomfortable frequency range $(0.8-1.6 \mathrm{~Hz})$ is suppressed by the proposed method.

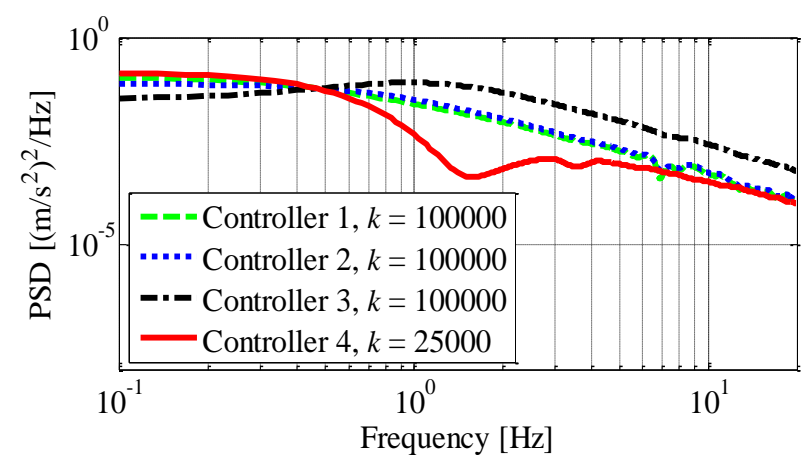

(A) Horizontal direction

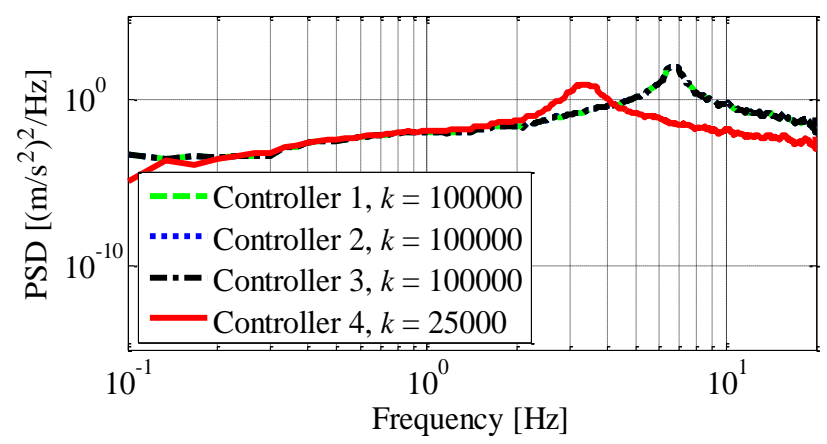

(B) Vertical direction

Fig. 10. PSD of a rider's head acceleration (Random road, Level C)

Fig. 11(A) shows that the RMS value of Controller IV is $13 \%$ smaller than that of Controller 2 which has the same settling time as Controller 4.

In Fig. 9(B), the proposed method suppresses the vertical head acceleration and makes the frequency of vibration lower. In Fig, 10(B), the peak of PSD moved from $6.7 \mathrm{~Hz}$ to $3.4 \mathrm{~Hz}$, the natural frequency after tire changing. In Fig. 11(B), Controller 4 cannot suppress the RMS value. However, if the tire with the desired stiffness is combined with Controller 4, the RMS value is about $79 \%$ smaller than the value of others.

From these results, in both directions, the effectiveness of the proposed method was confirmed.

\section{CONCLUSION}

In this paper, we evaluated ride comfort of an inverted pendulum personal mobility (PM) and proposed a method to improve it. First, we suggested a model limited to two dimensions, horizontal and vertical, considering the tire stiffness. We assumed that the PM runs on a sinusoidal or random road and evaluated the horizontal and vertical vibration of the head of the occupant in numerical simulations. The evaluation methods are frequency analysis using PSD and RMS. We set the evaluation standard in accordance with ISO 2631-1. We proposed improvement methods for each direction. In the horizontal direction, we proposed frequency shaped LQG control. In the vertical direction, analysis showed that it is difficult to improve on the control system design approach. Therefore we proposed a change the tire as a hardware approach. Using both methods simultaneously, in the 
Horizontal direction, we reduced the vibration of the frequency range $(0.8-1.6 \mathrm{~Hz})$ in which human feels uncomfortable. At one time, in the vertical direction, the peak of PSD moved from 6.7 Hz, including within the uncomfortable frequency range $(4.0-8.0 \mathrm{~Hz})$, to $3.4 \mathrm{~Hz}$, the natural frequency after tire changing. The results show that the proposed method was effective.

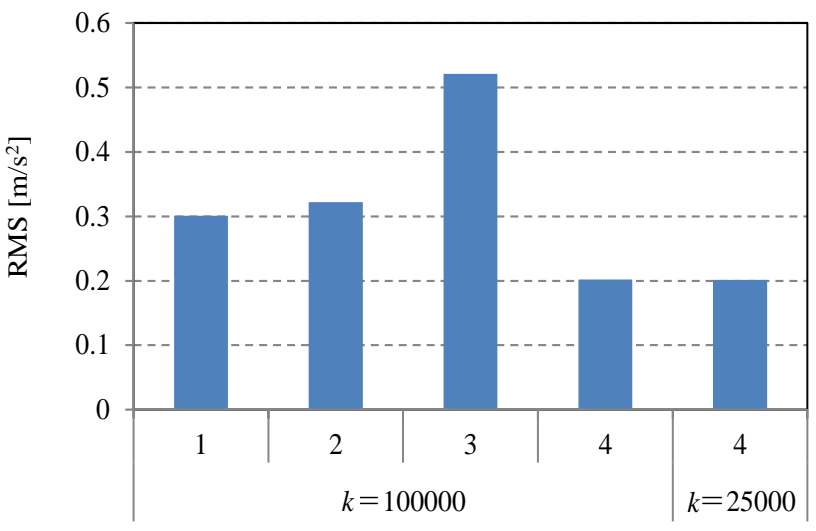

(A) Horizontal direction

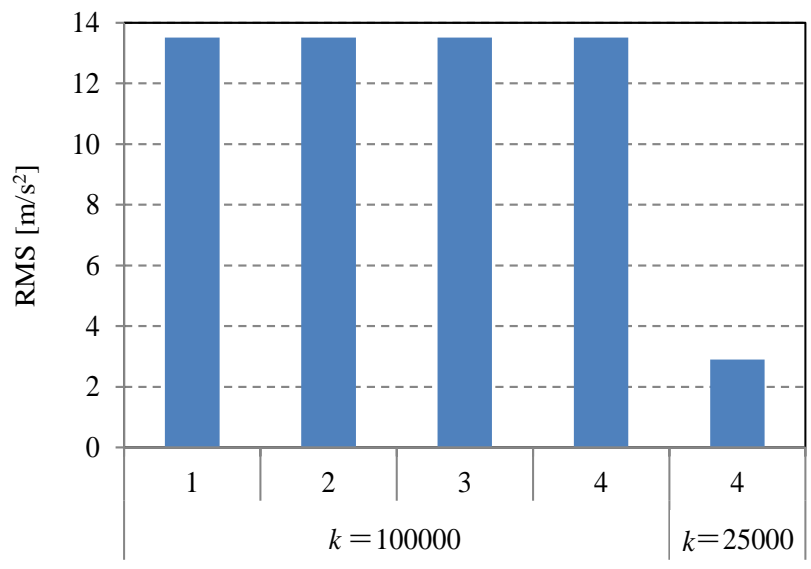

(B) Vertical direction

Fig. 11. RMS value of a rider's head acceleration (Random road, Level C)

As future works, we try to verify the validity of our proposed method through the simulations using the detailed model of occupant and the experiments using our developed inverted pendulum-type personal mobility.

\section{REFERENCES}

[1] Ministry of Internal Affairs and Communications, 2011. "The 2010 Census".

[2] S. Arakawa, C. Nakagawa, A. Shintani and T. Ito, "Basic Study on the Behavior of Inverted Pendulum Vehicle and Driver Using Multibody Dynamics", Transactions of the JSME, vol. 78, no.789, pp.1497-1506, 2012 (in Japanese).

[3] S. Ajisaka, T. Kubota and H. Hashimoto, "Human Balance Control Ability for Affinitive Personal Vehicle", 10th International Conference on Ubiquitous Robots and Ambient Intelligence (URAI), pp. 503-508, 2013

[4] C. Nakagawa, K. Nakano, Y. Suda and R. Hayashi, "Stability of the Dynamically Stabilized Two-Wheeled Vehicle Traveling on a Rough Road", Journal of Mechanical Systems for Transportation and Logistics, vol. 2, no. 1, pp. 78-89, 2009.

[5] F. Grasser, A. D.Arrigo, S. Colombi and A. C. Rufer, "JOE: a Mobile, Inverted Pendulum", IEEE Transactions on Industrial Electronics, vol. 49, no.1, pp. 107-114, 2002.

[6] G. C. Guerrero, J. J. R. Pasaye, "Real-time Control to Hold Upright Equilibrium of a Two-wheeled Auto-balancing Vehicle", Power, Electronics and Computing (ROPEC), IEEE International Autumn Meeting on Power, Electronics and Computing (ROPEC), pp. 1-5, 2013.

[7] J. Huang, F. Ding, T. Fukuda, T. Matsuno, "Modeling and Velocity Control for a Novel Narrow Vehicle Based on Mobile Wheeled Inverted Pendulum," IEEE Transactions on Control Systems Technology, vol. 21, no. 5, pp. 1607-1617, 2013.

[8] L. Zhijun, Y. Chenguang, "Neural-Adaptive Output Feedback Control of a Class of Transportation Vehicles Based on Wheeled Inverted Pendulum Models", IEEE Transactions on Control Systems Technology, vol. 20, Issue 6, pp. 1583-1591, 2012.

[9] Segway Japan, Ltd., "Home Segway Japan", segway-Japan.net. See also URL http://www.segway-japan.net/index.html, (accessed Jan, 2014).

[10] Ministry of Education, Culture, Sports, Science and Technology, "Investigation of Physical Fitness and Exercise Capacity". See also URL http://www.mext.go.jp/b_menu/toukei/chousa04/tairyoku/1261241.html, (accessed Jan, 2014)

[11] R. S. Sharp, S. Evangelou, D. J. N. Limebeer, "Advances in the Modeling of Motorcycle Dynamics", Multibody System Dynamics, vol. 12, no. 3, pp. 251-283, 2004.

[12] International Organization for Standardization ISO 8608, Mechanical vibration -Road surface profiles - Reporting of measured data, 1995.

[13] International Organization for Standardization ISO 2631-1, Mechanical vibration and shock - evaluation of human exposure to whole-body vibration - part 1: general requirements, 1997.

[14] L. Zuo, S. A. Nayfeh, "Low order continuous-time filters for approximation of the ISO 2631-1 human vibration sensitivity weightings", Journal of Sound and Vibration, 265, pp. 459-465, 2003.

[15] N. K. Gupta, "Frequency-Shaped Cost Functionals: Extension of LinearQuadratic-Gaussian Design Methods", Journal of Guidance, Control and Dynamics, vol. 3, no. 6, pp. 529-535, 1980.

[16] Sakai, H., 1987. Tire Engineering, Grand Prix, 1-32 Kanda Jinbo-cho, Chiyoda-ku, Tokyo (in Japanese).

[17] Kinetic Characteristics of Motorcycles and Environment Surrounding It, Society of Automotive Engineers of Japan, 1997. 